\title{
Les facteurs de satisfaction et d'insatisfaction aux cycles supérieurs dans les universités québécoises francophones
}

\author{
Brigitte Gemme \\ Université du Québec à Montréal \\ Yves Gingras \\ Université du Québec à Montréal
}

\begin{abstract}
RÉSUMÉ
Mieux connaître les facteurs qui déterminent le niveau de satisfaction des étudiantes et étudiants en formation à la recherche (maîtrise et doctorat) peut nous aider à comprendre les causes du taux élevé de non-diplomation ce palier du système d'éducation. Sur la base d'un questionnaire administré à près de mille personnes étudiant ou ayant étudié aux cycles supérieurs dans les universités québécoises francophones, cet article présente les variables qui affectent le niveau de satisfaction globale des étudiantes et étudiants à l'égard de leur expérience dans leur programme d'étude. Il ressort clairement que les facteurs associés à la socialisation au métier de chercheur, soit principalement l'encadrement (notamment par la directrice ou le directeur), les progrès dans la production de résultats de recherche et la publication de ces derniers, sont des déterminants importants du niveau de satisfaction. Le type de financement obtenu a aussi un effet significatif, tandis que le sexe et l'âge ne sont pas des variables déterminantes de la satisfaction. Le succès de la formation à la maîtrise et au doctorat semble donc passer, au moins en partie, par une socialisation encadrée aux différents aspects du métier de chercheur.
\end{abstract}




\begin{abstract}
Elucidating the factors that determine the level of satisfaction of graduate students may help us explain the high attrition rates observed in master's and doctoral programs. Based on a survey of nearly one thousand students and graduates of master's degree and $\mathrm{PhD}$ programs in Québec's francophone universities, this paper examines the variables affecting their overall satisfaction from their studies. The findings suggest that the factors most closely related to research training are strongly associated with satisfaction. These factors include supervision and the capacity to produce and publish research results. Moreover, the type of funding secured by students is significantly associated with global satisfaction, while gender and age are not. The study concludes that supervised socialization into the role of professional researcher contributes to students' success in graduate programs.
\end{abstract}

Si l'acquisition des savoirs constitués d'une discipline ou d'une pratique professionnelle constitue le plus souvent l'objet des programmes d'études de premier cycle, les programmes des cycles supérieurs, pour leur part, visent plutôt le développement des compétences en recherche qui permettront aux diplômés de contribuer eux-mêmes à l'avancement des connaissances. Au cours de cette longue période de formation, les étudiantes et étudiants participent, à divers degrés, à un processus de socialisation professionnelle au terme duquel ils doivent avoir acquis l'habitus correspondant à leur domaine d'expertise. Or, il est connu qu'un nombre important des aspirants-chercheurs n'obtiennent pas le diplôme du programme auquel ils sont inscrits : en 2001-2002, parmi tous ceux qui sont sortis d'un programme de maîtrise, 70,4 \% l'ont fait avec un diplôme en main, tandis que cette proportion se situait à 53,0 \% au doctorat (Ministère de l'Éducation du Québec, 2004, p. 84 et 86)..$^{1}$ On sait aussi que le taux de réussite varie considérablement d'un domaine d'études à l'autre, les disciplines des sciences naturelles présentant de meilleures statistiques que les sciences humaines (Association canadienne pour les études supérieures, 2003; Bowen \& Rudenstine, 1992). Par exemple, les données présentées par l'Association canadienne pour les études supérieures (2003) indiquent que le taux de diplomation au doctorat dans les humanités est d'environ $45 \%$ tandis qu'il se situe au-delà de $70 \%$ dans les sciences biologiques.

Quels sont les facteurs de risque qui diminuent les chances de réussite du projet de formation à la recherche? C'est la question à laquelle nous tenterons d'apporter des pistes de réponse grâce aux résultats d'une enquête par questionnaire réalisée ayant rejoint près de 1000 personnes étudiant ou ayant étudié aux cycles supérieurs (maîtrise et doctorat) dans une université québécoise. Dans cet article, nous présenterons d'abord la littérature sur l'expérience étudiante à la maîtrise et au doctorat en nous attardant plus 
particulièrement aux facteurs qui ont déjà été identifiés comme favorables ou défavorables à la réussite. Ensuite, nous présenterons brièvement l'enquête que nous menons sur la formation à la recherche au Québec et la méthodologie employée pour l'analyse des facteurs de satisfaction et d'insatisfaction. Nous examinerons ces facteurs avant de conclure avec une discussion des résultats, des pistes de recherche à privilégier pour l'avenir et des actions à envisager pour favoriser la formation des jeunes chercheurs.

\section{LES FACTEURS DE RÉUSSITE AUX CYCLES SUPÉRIEURS}

$\mathrm{Au}$-delà du processus scolaire par lequel les étudiantes et étudiants cheminent pour obtenir un diplôme, la formation à la maîtrise (dans le profil recherche à tout le moins) et au doctorat constitue le mécanisme par lequel les inscrits doivent passer de l'acquisition à la production de connaissances. Pour plusieurs, cette transition est ardue car il s'agit désormais de répondre à des exigences très différentes de celles qui prévalaient dans les paliers antérieurs du système scolaire (Lovitts, 2005). Il ne faut donc pas se surprendre que la qualité du dossier universitaire, mesurée principalement par les notes ou les résultats à des épreuves uniformes comme le Graduate Record Examination (GRE), soit sans lien avec la persévérance effective aux études supérieures (Erhenberg \& Mavros, 1995; Lovitts, 2001).

Dans l'enquête de Lovitts, qui s'est intéressée à 18 départements de deux universités américaines, il est clairement démontré que le fait de mener à terme ses études doctorales ne résulte pas tant de l'aptitude académique (mesurée par les notes ou même par les publications antérieures) que du niveau d'intégration sociale et surtout académique des étudiantes et des étudiants. Ceux qui bénéficient de soutien institutionnel, notamment sous la forme de financement et d'un lieu de travail, qui sont encadrés par une directrice ou un directeur encourageant et qui évoluent dans un climat positif où ils sont entourés de pairs traversant les mêmes épreuves ont beaucoup plus de chances de compléter leur doctorat que ceux qui n'ont pas accès à ces ressources. Cet effet s'explique par le plus grand nombre d'occasions que ces étudiantes et étudiants ont de développer et d'enrichir la "carte cognitive " nécessaire à leur intégration et à leur persévérance. Cette " carte cognitive " serait en fait la perspective collective propre aux membres de chaque champ, laquelle donne un sens aux différentes étapes à franchir et l'orientation nécessaire pour prendre des décisions éclairées. Dans le cas des étudiantes et étudiants aux cycles supérieurs, cette carte faciliterait donc la progression dans le programme. À l'inverse, les étudiants qui n'ont que peu ou pas d'opportunités d'intégration deviennent plus insatisfaits à l'égard de leur programme, des membres du corps professoral, de leur directrice ou de leur directeur et de leurs pairs. Ils se sentent davantage isolés et sont moins attachés au milieu académique. En plus de prendre de mauvaises décisions résultant d'un manque d'information et de se désengager de leurs études, avec des conséquences parfois importantes pour leur succès 
scolaire, ces personnes à risque deviennent plus vulnérables aux perturbations venues de l'extérieur, comme des difficultés financières ou familiales. Lovitts résume bien le processus :

Thrown back on their individuality and their own resources, they come to see themselves as figures against a ground of which they are not a part. Their actions and interactions become effortful. As a result, their performance suffers, and they start to question whether they are achieving their goals, examine their reasons for being a member of that community, and assess whether the benefits of persistence are worth the costs. (p. 262)

En ce sens, l'explication apportée par Lovitts rejoint le point de vue plus théorique de Tinto (1993) sur la formation aux cycles supérieurs : bien qu'au premier cycle l'intégration sociale soit aussi importante que l'intégration académique, cette dernière devient nettement dominante à la maîtrise et au doctorat, où la socialisation au métier de la recherche est l'objet même de la formation. Les variables importantes à examiner ne sont donc pas tant institutionnelles, c'est-à-dire liées à l'université d'attache de l'étudiante ou de l'étudiant. Elles se situent plutôt à l'échelle départementale et, plus encore, au niveau de l'entourage immédiat de la personne en formation, où la directrice ou le directeur occupe une place prépondérante. De plus, les disciplines étant le principe de l'organisation du monde académique, il s'agit, largement, d'une socialisation disciplinaire (Gingras, 1991; Becher \& Trowler, 2001). ${ }^{2} \mathrm{Ce}$ processus de socialisation, s'il est réussi, devrait déboucher sur l'acquisition par l'apprentie-chercheuse ou l'apprenti-chercheur de l'habitus approprié, c'est-àdire de l'ensemble des schèmes d'action et de perception des pratiques légitimes acquis au cours de la formation (Kaufmann, 2000). Défini ainsi, l'habitus est très proche du concept de carte cognitive utilisé par Lovitts, tout en ayant sur ce dernier l'avantage de se centrer sur les pratiques, plus directement observables. Le contenu de cet habitus est toutefois diffıcile à définir. Suite à de nombreuses entrevues avec des professeures et professeurs dirigeant des thèses, Delamont, Parry et Atkinson (2000) concluent qu'il n'y a guère de consensus entre les disciplines et même à l'intérieur de celles-ci sur ce qui fait la qualité du travail étudiant. Cependant, trois aspects ressortent de manière relativement constante : l'originalité, la capacité de recueillir des données appropriées et l'aptitude à inscrire sa recherche dans le cadre des savoirs existants. Au-delà des compétences techniques que cela suppose, il s'agit essentiellement de " compétences indéterminées " (indeterminate skills) que les personnes qui dirigent et évaluent les thèses savent reconnaître, mais qu'elles ne peuvent expliciter. En fait, l'acquisition de cette capacité de jugement est justement au cœur de la formation doctorale : tant dans les sciences naturelles que dans les sciences sociales et humaines, l'apprentissage de la frontière entre ce qui " marche " et ce qui ne " marche pas ", qu'il s'agisse d'une expérience ou d'une 
analyse, est crucial. C'est en faisant de la recherche et en la confrontant aux autres travaux passés ou en cours de sa discipline que l'étudiante ou l'étudiant acquiert ces compétences. Or, les conditions de cette recherche sont très différentes d'une discipline à l'autre et ont des impacts importants sur la socialisation des apprentis. Le travail de laboratoire, par exemple, offre davantage d'occasions de socialisation que le travail de terrain, au cours duquel les étudiantes et étudiants sont bien souvent isolés (Roth \& Bowen, 2001; Delamont, Parry \& Atkinson, 2000).

Dans tous les cas, l'interaction avec la chercheuse ou le chercheur expérimenté qui dirige le projet de recherche est cruciale et l'encadrement offert détermine en bonne partie l'intégration académique de l'étudiante ou de l'étudiant. Le contraste est même assez marqué entre deux catégories de professeurs : dans l'enquête de Lovitts (2001), les étudiants qui avaient quitté leur programme de doctorat et les étudiants à risque (qui avaient songé à abandonner) n'avaient pas eu le même type de directeur que les autres étudiants persévérants. En effet, Lovitts a d'abord constitué un échantillon de professeurs divisé en deux groupes : les "grands producteurs de Ph.D. ", c'est-à-dire les professeurs ayant supervisé plusieurs diplômés de leur département, et les "faibles producteurs ", soit ceux ayant supervisé seulement quelques-uns des diplômés. Elle les a ensuite interviewés pour comprendre leur approche respective de la supervision d'étudiant. Les "faibles producteurs " auraient moins tendance à prendre l'initiative des contacts ou à offrir de l'information ou des conseils à leurs protégés, seraient moins investis (académiquement et socialement) envers leurs étudiants ou envers les activités de leur département en général, et moins impliqués dans la recherche de pointe. Au contraire, les "grands producteurs " auraient plutôt l'habitude de contribuer spontanément à la socialisation de leurs étudiants en s'assurant d'une relation plus soutenue, non seulement dans le strict cadre de la thèse mais aussi à travers la participation à d'autres activités départementales, académiques ou sociales. Ils seraient également plus actifs en recherche en général et nettement plus susceptibles d'inciter leurs protégés à participer à des conférences, à publier des articles (notamment en collaboration avec eux) et à s'impliquer dans les organisations professionnelles de leur discipline.

Ainsi, Lovitts constate que les diplômés et les persévérants ont nettement plus tendance à décrire leur directrice ou leur directeur en des termes qui associent ces derniers au profil des "grands producteurs " et beaucoup plus nombreux à être satisfaits de leur relation avec ce dernier que les autres. Ces résultats de l'enquête de Lovitts concordent avec ceux, plus anciens, de Pease (1967), ce qui démontre la pérennité du phénomène : ce dernier notait déjà que la multiplication des contacts entre les étudiants et les membres du corps professoral était corrélée avec une socialisation professionnelle accrue des étudiants, notamment une plus grande participation à la publication d'articles et l'établissement de plus nombreux contacts professionnels dans leur champ d'études. 
La qualité de l'encadrement offert par la directrice ou le directeur est un facteur crucial dans la réussite de la socialisation des jeunes chercheurs mais ce n'est évidemment pas le seul. Le financement est également une ressource importante pour la réussite du projet d'études. Le niveau de financement n'est toutefois pas la seule variable en jeu : on observe généralement que les modes de financement les plus associés à l'obtention du diplôme sont ceux qui favorisent un meilleur encadrement des études, en particulier les emplois d'assistante ou d'assistant de recherche (Ehrenberg \& Mavros 1995). Lovitts (2001) observait pour sa part que les auxiliaires d'enseignement semblent mieux intégrés dans leur département que les étudiantes et étudiants qui obtiennent une bourse sans obligation de travailler. Cet effet est plus marqué quand les auxiliaires doivent participer à des séances d'information et de formation avant de commencer à enseigner, en plus de partager un lieu de travail avec leurs pairs. Bowen et Rudenstine (1992) ont quant à eux été surpris de constater que les prestigieux programmes de bourses d'excellence disponibles pour les meilleurs étudiants américains n'accroissent pas significativement les chances de réussite des boursiers s'ils ne s'accompagnent pas de mesures d'encadrement adéquates. L'accès au financement est donc une aide à la réussite seulement dans la mesure où il est aussi un vecteur de socialisation, c'est-à-dire quand il favorise la création et l'intensification des liens entre l'étudiante ou l'étudiant et sa communauté académique.

D'autres éléments peuvent favoriser ou nuire à la socialisation, dont plusieurs dépendent du département au sein duquel l'étudiante ou l'étudiant est inscrit. Bowen et Rudenstine (1992) soulignent notamment que les grands départements, qui hébergent de nombreux étudiants, sont moins propices à la réussite que les petits départements. Cette étude suggère que, à l'inverse, les départements ayant mis sur pied un programme d'études structuré et cohérent favorisent la réussite étudiante. La culture départementale est également un facteur déterminant pour l'insatisfaction et la non-persévérance étudiante aux cycles supérieurs : un département au climat malsain (caractérisé, du point de vue étudiant, par la discrimination ou le harcèlement sexuel par exemple) ou ne permettant pas l'établissement de liens interpersonnels est propice à l'abandon des études (Lovitts, 2001).

Enfin, il faut noter que certaines situations dans lesquelles se retrouvent, volontairement ou non, les étudiantes et les étudiants peuvent également nuire à leur intégration académique. Ceux qui vivent des conflits de rôle, notamment parce qu'ils ont des engagements importants à l'extérieur de l'université comme des responsabilités familiales ou un emploi, peuvent avoir des difficultés à s'intégrer (Tinto, 1993 ; Deem \& Brehony, 2000), tout comme ceux qui suivent leur programme à temps partiel, les étudiants étrangers et ceux qui n'aspirent pas à une carrière universitaire (Deem \& Brehony, 2000). Dans une étude canadienne, Seagram, Gould et Pyke (1998) ont également observé que les femmes seraient plus susceptibles d'être insatisfaites de leur expérience de formation. D'après Legault (1997), les étudiantes et les étudiants à la maîtrise 
exprimeraient aussi davantage d'insatisfaction à l'égard de leurs études que ceux au doctorat, possiblement en raison des tâches moins enrichissantes qui leur sont confiées.

Il ressort donc nettement des travaux antérieurs sur la formation à la recherche que la socialisation académique, qui est en fait une sorte de socialisation professionnelle, est au cœur de l'expérience étudiante aux cycles supérieurs. Son bon déroulement est nécessaire à la réussite des études de maîtrise et de doctorat. Nous avons voulu vérifier, au sein d'un échantillon de personnes étudiant ou ayant récemment étudié aux cycles supérieurs au Québec, le poids des différents aspects de la socialisation à la recherche dans leur satisfaction à l'égard de leur expérience globale de formation. Tous nos répondants sont des persévérants, c'est-à-dire que tous poursuivent leurs études ou les ont terminées avec succès. Il est toutefois probable qu'un certain nombre des répondants, toujours inscrits au moment de l'enquête, n'obtiendront pas le diplôme de leur programme. Notre enquête n'ayant pas à l'origine été conçue pour analyser les abandons scolaires, nous n'avons pu recueillir le témoignage de personnes ayant quitté leur programme d'études afin d'expliquer directement leur départ. ${ }^{3}$ Néanmoins, nous savons, depuis les travaux de Seymour et Hewitt (1997) portant sur le départ des programmes scientifiques au premier cycle, que les insatisfactions qui poussent certaines personnes à abandonner leur programme leur sont rarement exclusives : celles qui persévèrent partagent souvent ces insatisfactions, sans toutefois que cela n'entraîne l'abandon des études. Lovitts (2001) a également observé d'importantes similarités entre les étudiantes et étudiants persévérants mais " à risque " et celles et ceux ayant abandonné leur programme. L'examen des sources d'insatisfaction des persévérants et des diplômés devrait contribuer à l'identification, sinon des motifs de départ, au moins des facteurs de risque susceptibles de mener à l'interruption de la formation à la recherche. En se limitant aux persévérants et aux diplômés, notre approche complémente par ailleurs les travaux qui portent exclusivement sur les étudiantes et étudiants ayant quitté leur programme (Golde, 1998).

\section{MÉTHODOLOGIE}

Cette analyse des facteurs d'insatisfaction des étudiantes et des étudiants aux cycles supérieurs s'inscrit dans une recherche plus vaste portant sur l'ensemble de la formation à la recherche. Cette recherche vise une meilleure compréhension de l'expérience étudiante aux cycles supérieurs dans le contexte des transformations des pratiques, en particulier de l'accroissement des interactions université-milieu (avec l'industrie, les gouvernements ou le milieu communautaire et associatif).

Dans ce contexte, un questionnaire comportant environ 150 questions, la plupart à choix multiples, a été administré par Internet. ${ }^{4}$ Le questionnaire a été construit à la lumière de la littérature disponible et d'une série d'entretiens exploratoires réalisés avec des étudiants, des professeurs et des administrateurs universitaires. Il aborde les principales dimensions de l'expérience étudiante 
en formation à la recherche, soit : la trajectoire dans le programme d'études, les lieux de travail et d'études, le domaine d'études, la discipline et le sujet du mémoire ou de la thèse, le contexte plus vaste du projet (s'il y a lieu), les personnes impliquées dans le projet ou offrant du soutien, l'encadrement (en particulier la relation avec la directrice ou le directeur), le financement, les autres ressources, les résultats de recherche (notamment les publications) et un bilan de l'expérience (incluant notamment le niveau de satisfaction). Lorsque c'était pertinent, des questions ont également été posées sur les relations avec des organisations non-universitaires (entreprises, gouvernement, organisations sans but lucratif, etc.). De plus, nous avons posé aux personnes qui étaient encore aux études des questions sur leurs projets professionnels tandis que les personnes déjà diplômées ont dû répondre à des questions portant sur leur insertion professionnelle. ${ }^{5}$

Le questionnaire a été rempli par près de 1000 répondants entre le printemps 2003 et l'été 2004. ${ }^{6}$ Afın de favoriser la participation de répondantes et de répondants aussi diversifiés que possible, nous avons emprunté plusieurs voies de recrutement. Les principales universités francophones québécoises offrant des programmes de formation à la recherche ont effectué des envois par courrier électronique à l'ensemble de leur population étudiante aux cycles supérieurs. De plus, les associations étudiantes ainsi que certaines associations de diplômés ont sollicité leurs membres par courrier électronique et des brochures ont été distribuées et affichées dans la plupart des institutions. Les organismes subventionnaires québécois ont également contribué à l'enquête en envoyant à l'ensemble des boursiers des cinq dernières années une invitation à participer à l'enquête. De plus, nous avons constitué des listes d'envoi électroniques spécifiques à notre projet de recherche pour favoriser le recrutement de répondants participant à des projets de recherche réalisés en collaboration avec le milieu industriel, gouvernemental ou associatif. ${ }^{7}$

$\mathrm{Au}$ total, 956 questionnaires complets ont été recueillis. On trouve 45 $\%$ d'hommes et $55 \%$ de femmes parmi nos répondants. ${ }^{8}$ Les populations étudiantes des différentes universités francophones y sont représentées de manière relativement équilibrée au sein de l'échantillon, à l'exception de ceux de l'Université de Montréal qui sont un peu plus nombreux qu'attendu en fonction de leur poids réel dans la population étudiante aux cycles supérieurs du Québec. Notre enquête ne permet toutefois pas de bien représenter la situation des étudiantes et des étudiants inscrits à temps partiel car ceuxci ne composent que $6 \%$ de notre échantillon. ${ }^{9}$ De plus, les boursiers des organismes subventionnaires québécois sont également surreprésentés en raison des paramètres de la première partie de l'enquête (qui visait spécifiquement les boursiers en milieu de pratique) et de l'envoi électronique effectué par les organismes subventionnaires. On compte un minimum de 304 boursiers confirmés au sein de notre échantillon (31,8 \%) et 161 autres répondants (16,8 $\%$ ) ont affirmé avoir bénéficié d'une bourse d'un organisme subventionnaire québécois ou canadien sans qu'il soit possible de vérifier leur statut. Au total, 
donc, presque une personne sur deux dans notre échantillon aurait obtenu une bourse d'excellence. Cette proportion est nettement supérieure à celle qui prévaut dans la population étudiante, dans laquelle on compte, au total, un peu moins de $30 \%$ de récipiendaires de bourses d'excellence, quelle que soit leur nature, dont approximativement la moitié reçoit une bourse d'un organisme subventionnaire québécois ou canadien (Conseil national des cycles supérieurs, 2001). Notre échantillon est donc constitué de nombreuses personnes qui ont une situation relativement avantageuse dès le début de leurs études : elles sont inscrites à temps plein et bénéficient de bourses d'études. Ces caractéristiques de nos répondantes et répondants permettent ainsi d'examiner une population potentiellement privilégiée et d'apporter un éclairage original sur la question de la persévérance à la maîtrise et au doctorat.

Enfin, nous avons limité l'analyse de ces données à la strate de répondantes et répondants qui ont au moins débuté leur processus de recherche. Nous avons donc exclu les répondants qui, depuis le début de leur programme, avaient seulement suivi les cours de leur scolarité à l'exclusion de toute recherche ou rédaction. Nous avons cependant conservé dans cette strate les répondants qui sont à la fois en scolarité et en recherche, une situation fréquente en sciences en particulier. Ainsi, seuls 841 des 956 répondantes et répondants ont été retenus pour l'analyse présentée ici.

L'échantillon sur lequel se basent nos résultats de recherche n'est donc pas statistiquement représentatif de la population étudiante aux cycles supérieurs québécois. Cependant, la diversité des situations étudiantes qui y sont représentées rend tout à fait possible l'examen des relations entre certaines caractéristiques de l'expérience étudiante et le niveau de satisfaction des personnes sondées.

Afın de découvrir quelles dimensions de l'expérience de formation à la recherche influencent le niveau de satisfaction des étudiantes et étudiants, nous avons effectué des analyses bivariées entre chacune de nos variables et le niveau de satisfaction (recodé numériquement). Nous considérons le niveau de satisfaction comme la variable dépendante à expliquer et toutes les autres variables comme des variables indépendantes potentiellement explicatives. Nous avons appliqué le test t de Student pour identifier quelles modalités de chaque variable présentent une différence significative par rapport à la moyenne (au risque de $5 \%$ ).

Lorsque cela permettait de clarifier l'exposé des résultats, nous avons aussi effectué des tris croisés entre le niveau de satisfaction (sous forme de modalités discrètes et non d'échelle numérique) et les modalités d'autres variables. Dans ces cas, le test $d u \chi^{2}$ a permis de vérifier le caractère significatif des liens entre les variables (au risque de $5 \%$ ). Les tableaux 2 et 3 sont des exemples de ces analyses. Enfin, quand les variables indépendantes le permettaient, c'est-à-dire lorsqu'il s'agissait aussi d'une variable en échelle ou numérique, nous avons également effectué des corrélations et des régressions.

Dans cet article, sauf mention explicite, nous présentons exclusivement les variables pour lesquelles un lien significatif est observé entre certaines 
modalités (représentant une situation en formation à la recherche) et le niveau de satisfaction déclaré des répondants. Le tableau 4 présente l'effet de toutes les variables pertinentes de manière synthétique.

\section{RÉSULTATS}

Nous avons mesuré la satisfaction des étudiantes et étudiants sur la base de la question suivante : "Quel est votre niveau de satisfaction globale par rapport à vos études de maîtrise ou de doctorat ? " Les choix suivants étaient offerts : " Très insatisfait ", " Plutôt insatisfait ", " Plutôt satisfait " et " Très satisfait ". Comme on peut le voir au tableau 1, la majorité des répondants $(51,0 \%)$ sont "plutôt satisfaits " et seuls 14,2 \% sont "plutôt " ou " très insatisfaits ". Nous avons aussi recodé numériquement ces choix de réponse, "Très insatisfait " devenant 1 et "Très satisfait " devenant 4 . Le niveau de satisfaction moyen est donc 3,16 sur 4 (avec un écart-type de 0,76).

\section{Tableau 1}

Distribution des niveaux de satisfaction globale des étudiantes et étudiants aux cycles supérieurs

\begin{tabular}{lcc}
\hline Niveau de satisfaction & $\mathrm{N}$ & $\%$ \\
\hline Très insatisfait & 33 & 3,9 \\
Plutôt insatisfait & 87 & 10,3 \\
Plutôt satisfait & 429 & 51,0 \\
Très satisfait & 287 & 34,1 \\
Total & 841 & 100,0 \\
\hline
\end{tabular}

\section{Les caractéristiques à l'entrée}

Les caractéristiques personnelles des étudiantes et des étudiants sont-elles liées à la manière dont l'expérience du programme est vécue ? Au sein de notre échantillon, cet effet est faible. Le sexe des répondants n'est pas corrélé avec leur niveau de satisfaction à l'égard de leur expérience de formation. L'appartenance à un groupe d'âge spécifique n'est pas non plus corrélée avec le niveau de satisfaction. Cependant, le fait d'avoir un enfant est associé à un taux plus bas d'insatisfaction : les répondants ayant un enfant sont environ trois fois moins susceptibles d'être " plutôt " ou " très insatisfaits " que ceux qui n'ont pas d'enfant ou qui en ont deux ou plus (4,5\% contre $14,9 \%$ pour ceux qui n'ont pas d'enfants et 17,5\% pour ceux qui en ont deux ou plus). ${ }^{10}$ En somme, s'il est presque certain que les variables sociodémographiques ont un impact sur la persévérance et la durée des études, elles n'en ont que peu sur la satisfaction générale des répondantes et répondants.

On aurait pu s'attendre à ce que les étudiantes et étudiants des différents domaines d'études présentent des différences quant à leur satisfaction. Or, nous 
n'avons cependant pas observé de lien significatif entre l'appartenance à l'un des trois grands groupes disciplinaires (sciences naturelles et génie, sciences de la santé, sciences humaines et sociales) et le niveau de satisfaction.

Une seule variable de ce groupe présente une relation nette avec le niveau de satisfaction : le cycle d'études. Les répondantes et répondants qui remplissent le questionnaire au sujet de leur programme de maîtrise sont deux fois plus susceptibles d'être insatisfaits de leur expérience de formation (19,1\% sont " très " ou "plutôt insatisfaits ") que ceux au doctorat $(9,8 \%)$. Cette association est statistiquement très significative. Comme nous l'avons vu, Legault (1997) fait l'hypothèse que cette insatisfaction est le fait des tâches moins diversifiées et spécialisées confiées aux étudiants à la maîtrise. Nous croyons aussi qu'il est possible que ces niveaux de satisfaction nettement différents soient le résultat d'un processus d'autosélection : les étudiantes et étudiants qui n'ont pas apprécié leur expérience de formation à la recherche à la maîtrise ont probablement moins de chances d'avoir poursuivi des études doctorales, ce qui expliquerait le taux relativement plus élevé de satisfaction chez ceux qui ont décidé de passer au troisième cycle. Enfın, comme nous le verrons plus bas, certains autres facteurs associés à l'insatisfaction affectent aussi les étudiantes et étudiants inscrits à la maîtrise de manière plus marquée.

\section{Le financement des études et les ressources disponibles}

La poursuite d'études aux cycles supérieurs peut s'avérer coûteuse, notamment pour celles et ceux qui, de fait ou par choix, ne disposent pas du soutien financier de leur famille. Le financement des études est d'ailleurs une préoccupation centrale des organisations étudiantes à cet ordre d'enseignement (Conseil national des cycles supérieurs, 2001). Au-delà du niveau de revenus dont disposent les étudiantes et étudiants pour subsister, on peut aussi interpréter les différentes sources de financement comme autant de signes de l'intégration sociale et académique. En effet, connaître les différentes sources de financement disponibles et poser sa candidature avec succès résulte d'un apprentissage qui ne va pas de soi car ces structures ne font pas partie de l'univers du premier cycle. Comme l'information à ce sujet est largement implicite, son acquisition repose sur l'intégration dans un réseau de pairs et de chercheurs établis. De plus, comme nous l'avons vu, certaines formes de financement, en particulier celles qui exigent un travail, offrent aussi un potentiel d'intégration supérieur. Ces formes de financement supposent une présence soutenue à l'université ainsi que, possiblement, un engagement plus grand envers la communauté académique.

Il n'est donc pas surprenant que certains modes de financement soient plus fortement associés à la satisfaction ou à l'insatisfaction que d'autres. On observe que les étudiantes et étudiants qui sont titulaires d'une bourse d'un organisme subventionnaire québécois ou canadien (FQRSC, FQRNT, FRSQ, CRSH, CRSNG, IRSC) sont nettement plus satisfaits de leur expérience que ceux qui n'en ont pas obtenu. Presque la moitié d'entre eux (43\%) se disent même 
" très satisfaits " de leur expérience, contre seulement $25 \%$ de ceux qui n'ont pas obtenu de financement de cette source. À un degré un peu moindre, les emplois d'assistant d'enseignement ou de recherche sont également associés à une satisfaction accrue de celles et ceux qui les occupent. A contrario, le fait de devoir s'endetter pour étudier est associé à plus d'insatisfaction : les bénéficiaires de l'Aide financière aux études (" prêts et bourses ") du ministère de l'Éducation du Québec sont deux fois plus nombreux à se dire "plutôt " ou " très insatisfaits " que ceux qui n'y ont pas recours (23\% contre $12 \%)$. Les étudiantes et étudiants à la maîtrise étant plus nombreux à recourir à l'aide financière du ministère que ceux du doctorat (36,6\% contre 12,6\%), il pourrait s'agir là d'une des explications de leur taux accru d'insatisfaction.

Quant au jugement que les répondantes et répondants portent sur le financement de leurs études, il est associé à la satisfaction en général : moins un étudiant est satisfait du financement obtenu pour ses études, moins il a de chances d'être satisfait en général. Néanmoins, bien que le test du $\chi^{2}$ effectué sur le tri croisé donne un résultat très significatif, la corrélation entre les deux variables est relativement faible. En effet, le niveau de satisfaction à l'égard du financement (lorsque converti en échelle de 1 à 4) n'explique que $5 \%$ de la variance de la satisfaction en général (coefficient de corrélation : +0,24).

À la fin de la section du questionnaire portant sur le financement des études, nous avons demandé aux répondants d'apporter des précisions sur leur situation s'ils le jugeaient nécessaire. Quand on observe en particulier les commentaires formulés par les répondants insatisfaits, on remarque une certaine dose d'exaspération, même si cette dernière est parfois résignée :

Pas une "maudite " cenne d'aide de mon directeur de recherche... (186)

J'ai des difficultés financières et j'ai besoin d'un ordinateur portable (exigé par mon encadreur) et je suis vraiment stressée ! (161)

J'ai décidé de faire cette maîtrise en dépit du [manque de] financement, or, trois ans, c'est long pour être pauvre. (11)

On remarque d'ailleurs que plusieurs étudiants font ici référence à leur directrice ou à leur directeur, généralement pour déplorer que celui-ci n'ait pas répondu à leurs attentes (ou même tenu ses propres promesses) d'aide financière.

Au-delà du financement, d'autres ressources matérielles peuvent être nécessaires à la réalisation du projet de l'apprentie-chercheuse ou de l'apprentichercheur. Au nombre de celles-ci, on compte un lieu de travail, un ordinateur, de l'équipement spécialisé ainsi que du soutien technique et administratif. Sans surprise, la mesure de la satisfaction spécifique à certains de ces items est associée au niveau global de satisfaction des répondants quand on effectue un tri 
croisé. Toutefois, le pouvoir prédicteur global est relativement faible : dans une régression multiple, les deux variables associées à la satisfaction générale, soit la satisfaction quant à l'espace de travail et au soutien technique, déterminent ensemble seulement $11,1 \%$ de la variance de la satisfaction générale $(R=0,33)$. On note d'ailleurs que le fait d'avoir ou non accès à un bureau de travail n'affecte pas le niveau de satisfaction générale.

\section{L'encadrement}

Le rôle de la directrice ou du directeur aux cycles supérieurs est central dans le processus de socialisation des étudiantes et des étudiants. Il n'est donc pas surprenant d'observer d'importants liens entre les différents aspects de la supervision et le niveau de satisfaction de l'étudiant ou étudiante.

Des effets se font sentir aussi loin que dans les facteurs de choix qui ont amené l'étudiant à s'associer à tel directeur plutôt qu'à tel autre. En effet, il semble que certaines décisions aient été mieux éclairées que d'autres : les étudiants qui n'ont pas tenu compte de la réputation de chercheur ou de l'expertise spécifique de ce dernier dans le domaine qui les intéressait sont moins susceptibles d'être satisfaits de leur expérience de formation. De même, ceux qui n'invoquent pas les qualités personnelles de leur directeur dans les facteurs ayant influencé leur choix sont également plus nombreux à être insatisfaits de leur situation.

La fréquence des échanges avec la directrice ou le directeur - quel que soit le mode de communication emprunté (en personne, par téléphone ou par courrier électronique) - est également associé à la satisfaction : lorsque les contacts ont lieu une fois par mois ou moins, l'étudiante ou l'étudiant est plus susceptible d'être insatisfait de son expérience tandis que ceux qui voient leur directeur une fois par semaine ou davantage sont nettement plus satisfaits. En général, les personnes au doctorat disent rencontrer leur directeur un peu plus souvent que celles à la maîtrise, ce qui pourrait en partie expliquer la moindre satisfaction de ces derniers. Il n'existe pratiquement aucun lien entre le contenu de ces échanges et la satisfaction, à une exception près : ceux qui disent discuter avec leur directeur de textes écrits par d'autres auteurs sont plus satisfaits que ceux qui n'ont pas de discussions de ce type. On peut y voir une marque de socialisation professionnelle, les apprentis étant initiés aux critères d'évaluation en vigueur dans la communauté académique par la discussion avec une chercheuse ou un chercheur expérimenté des travaux de recherche des autres. Fait à noter, les étudiants qui disent discuter de leur avenir professionnel avec leur directeur sont plus satisfaits que ceux qui n'abordent pas cette question, mais le lien n'atteint pas le seuil que nous avons établi (93\% au lieu de $95 \%$ ). Ce résultat serait néanmoins cohérent avec ceux obtenus par Deem et Brehony (2000) selon lesquels les étudiantes et étudiants qui envisagent une carrière académique auraient davantage accès à la "culture de la recherche " que les autres, et seraient ainsi plus satisfaits de leur expérience. En effet, il est plausible que celles et ceux qui s'identifient au modèle professionnel incarné par 
la personne qui supervise leurs recherches ont probablement plus de chances de discuter de leur éventuelle carrière avec cette dernière. C'est une hypothèse qu'il faudrait explorer plus à fond, dans le cadre d'entrevues par exemple.

C'est donc sans surprise qu'une corrélation parmi les plus fortes de notre enquête est observée entre la satisfaction à l'égard de la directrice ou du directeur et la satisfaction en général. La première, à elle seule, explique en effet $10 \%$ de la variance de la seconde, avec un coefficient de corrélation de $+0,33$. Plus largement, la satisfaction à l'égard de l'ensemble de l'encadrement intellectuel reçu est également associée au niveau de satisfaction en général, expliquant 15 $\%$ de la variance au sein de notre échantillon, avec un coefficient de corrélation de $+0,39$. Combinées dans une régression multiple, les deux variables expliquent 19,1\% de la variance de la satisfaction générale des étudiantes et des étudiants $(R=0,44)$. Évidemment, ces deux variables sont intimement liées, la directrice ou le directeur étant un acteur central de l'encadrement. Il ne faut toutefois pas négliger de considérer le rôle de l'ensemble des autres personnes susceptibles de fournir du soutien aux étudiants (autres professeurs, personnel administratif, pairs, etc.). Ces derniers jouent un rôle de soutien, lequel peut devenir déterminant en cas de difficultés avec la directrice ou le directeur.

Ici aussi, il semblerait que l'insatisfaction des étudiantes et étudiants soit le fait d'attentes déçues : "Je pensais être plus encadré, un peu comme tous les autres. Je suis laissé à moi-même, très déçu de cela " (186). Très peu de répondants, parmi ceux qui se disent globalement insatisfaits de leur expérience, ont formulé des commentaires positifs au sujet de l'encadrement, mais certains sont plus compréhensifs que d'autres :

L'état lamentable des bibliothèques et des universités en général, l'épuisement des professeurs, le manque de financement et d'encadrement me font comprendre pourquoi le taux d'abandon est si élevé, moi qui y songe, malgré ma très grande motivation au départ. (466)

Mêmelorsquel'encadrement esteffectivement offert, destensionsimportantes peuvent surgir entre l'apprenti et son mentor, faute de personnalités compatibles ou à la suite de désaccords majeurs. Cette situation n'est apparemment pas la norme mais, quand elle se produit, la balance du pouvoir n'est généralement pas entre les mains de l'étudiante ou de l'étudiant :

Les relations entre mon directeur et moi sont plutôt tendues, mais je n'ai pas le choix de demeurer avec lui pour des raisons financières.

Le directeur ou la directrice peut ainsi devenir le cerbère du monde académique et rendre l'accès à ce dernier pratiquement impossible. Cette situation, si elle est ressentie comme injuste par l'étudiant, en particulier si l'alliance avec le directeur ou la directrice n'est pas le résultat d'un véritable choix mutuel, peut devenir une source majeure d'insatisfaction. 
La progression de la recherche

L'allongement des études aux cycles supérieurs est un sujet qui retient beaucoup l'attention des chercheurs et des administrateurs universitaires. Il préoccupe également les étudiants. En effet, ceux qui étaient toujours aux études au moment de participer à notre enquête et qui se percevaient en retard par rapport aux pratiques en vigueur dans leur milieu sont significativement plus nombreux à se dire insatisfaits de leur expérience. La même relation est observée chez les diplômés qui jugent avoir terminé leurs études en retard.

Force est toutefois de constater que le facteur qui est le plus fortement associé à la satisfaction des étudiantes et des étudiants à l'égard de leur expérience est l'évaluation qu'elles et ils font de leurs résultats de recherche. Quand on leur demande quel est leur degré de satisfaction par rapport à l'avancement de leurs recherches de maîtrise ou de doctorat, ou dans le cas des diplômés, leur degré de satisfaction par rapport aux résultats de leurs recherches, l'effet est clair. Chez les personnes qui sont toujours aux études, 91,3 \% de ceux qui sont "plutôt " ou "très satisfaits " de l'avancement de leurs travaux sont aussi satisfaits de leur expérience en général, contre 57,9\% de ceux qui sont insatisfaits de leurs résultats (tableau 2). Chez les personnes diplômées, la tendance est encore plus nette : 97,6\% de ceux qui sont satisfaits des résultats obtenus sont aussi satisfaits de leur expérience en général, tandis que seulement 26,7 \% de ceux qui sont insatisfaits de leurs résultats sont satisfaits de leur expérience en général (tableau 3). Ces étudiantes et étudiants ayant tout de même obtenu le diplôme de leur programme (malgré des résultats de recherche qu'ils jugent peu satisfaisants), on aurait pu s'attendre à ce qu'ils soient davantage satisfaits de leur expérience au bout du compte. Nous avons combiné la variable relative à l'expérience des étudiants et celle relative à l'expérience des diplômés dans une seule variable puis vérifié la corrélation entre cette dernière et le niveau de satisfaction global. Nous obtenons ainsi le coefficient de corrélation le plus élevé de ceux observés dans cette enquête $(+0,50)$, le niveau de satisfaction à l'égard des résultats de recherche produits ou en production expliquant $24 \%$ de la variance du niveau de satisfaction générale. Ceci confirme que c'est bel et bien l'apprentissage de la recherche qui est au cœur de l'expérience étudiante aux cycles supérieurs : quand la recherche ne donne pas de résultats satisfaisants (du point de vue des étudiants à tout le moins) c'est toute l'expérience de formation qui s'en trouve affectée.

Dans un même ordre d'idées, on remarque que les répondantes et répondants qui affirment avoir rencontré des difficultés dans la réalisation de leur projet de recherche sont plus susceptibles d'être insatisfaits de leur expérience, en particulier lorsque ces difficultés n'ont pas été résolues et sont jugées majeures. Ces difficultés peuvent relever de la nature même du projet initialement déterminé :

Le projet de recherche n'était pas terrible en partant et il ne s'est pas amélioré en cours de route, ce qui fait que les résultats intéressants 
ont été très peu nombreux, ce qui a entraîné des conflits avec mon co-directeur. (740)

Pour ma part, j'avais des tâches précises à remplir, qui m'ont d'ailleurs demandé beaucoup de temps pour peu de résultats publiables. (152)

Tableau 2

Niveau de satisfaction globale en fonction de la satisfaction à l'égard de la progression des recherches (étudiantes et étudiants toujours inscrits)

\begin{tabular}{lcccc} 
& \multicolumn{2}{c}{ Satisfaction à l'égard de la progression de recherches } \\
\cline { 2 - 5 } Satisfaction globale & \multicolumn{2}{c}{ Insatisfaits } & \multicolumn{2}{c}{ Satisfaits } \\
\cline { 2 - 5 } & $\mathrm{N}$ & $\%$ & $\mathrm{~N}$ & $\%$ \\
\hline Insatisfaits & 56 & $42,1^{\text {a }}$ & 47 & 8,7 \\
Satisfaits & 77 & 57,9 & 496 & 91,3 \\
Total & 133 & 100,0 & 543 & 100,0 \\
\hline
\end{tabular}

a. $\chi$ significatif au risque de $5 \%$.

Tableau 3

Niveau de satisfaction globale en fonction de la satisfaction à l'égard des résultats de recherche (diplômées et diplômés)

\begin{tabular}{lrrrr}
\hline & \multicolumn{2}{c}{ Satisfaction à l'égard de la progression de recherches } \\
\cline { 2 - 5 } & \multicolumn{2}{c}{ Insatisfaits } & \multicolumn{2}{c}{ Satisfaits } \\
\cline { 2 - 5 } Satisfaction globale & $\mathrm{N}$ & $\%$ & $\mathrm{~N}$ & $\%$ \\
\hline Insatisfaits & 11 & $73,3^{\text {a }}$ & 3 & 2,4 \\
Satisfaits & 4 & 26,7 & 123 & 97,6 \\
Total & 15 & 100,0 & 126 & 100,0 \\
\hline
\end{tabular}

a. $\chi^{2}$ significatif au risque de $5 \%$

Le premier extrait en particulier est intéressant en ce qu'il laisse transparaître un certain sentiment d'extériorité à l'égard du projet de recherche : l'étudiant parle " du " projet de recherche et non du " sien ». Delamont, Parry et Atkinson (2001) observent que, même dans les domaines d'études où les sujets de mémoire ou de thèse sont largement déterminés par le directeur, les étudiantes et étudiants s'approprient ce choix et en parlent comme de "leur " problème de recherche. On peut donc penser qu'une telle identification au projet de recherche et à ses résultats serait le fait d'une socialisation réussie, alors qu'une mise à distance, par l'étudiant, de ses propres travaux de recherche dénoterait des difficultés 
importantes et un risque de désengagement total à l'égard de l'apprentissage de la recherche. C'est une piste qu'il conviendrait d'explorer davantage à l'aide d'une démarche qualitative.

\section{Participer à la production scientifique}

En plus d'interroger les répondantes et répondants sur l'évaluation subjective qu'ils font de leurs résultats de recherche, nous avons cherché à recueillir des données plus objectives sur leur production scientifique en tant que telle, notamment en termes de publication et de commercialisation des résultats de recherche. Si ce dernier mode de production est sans lien avec le niveau de satisfaction, la participation à la diffusion des résultats de recherche par la publication écrite ou orale est, elle, associée à la satisfaction.

On observe en effet que les étudiantes et étudiants qui affirment n'avoir participé à aucune forme de diffusion des résultats de la recherche (article, chapitre de livre, affiche, communication, rapport, etc.) sont moins satisfaits que les autres de leur expérience de formation, comme on peut le voir au tableau 4. À l'inverse, ceux qui sont auteurs (ou co-auteurs) au moins une fois sont nettement plus satisfaits, en particulier s'ils ont écrit ou participé à l'écriture d'un article ou d'un rapport. De plus, parmi ceux qui ont déjà publié des résultats de recherche, on note que ceux qui ont vécu au moins une fois l'expérience de l'évaluation par les pairs sont plus satisfaits que les autres. L'évaluation par les pairs étant un aspect central du fonctionnement du monde académique, on peut penser que cette expérience est un élément important de la socialisation des apprentis-chercheurs. Elle vient confirmer, chez ceux dont l'article est accepté pour publication, l'appartenance à la communauté et donc la valeur des efforts investis jusqu'à présent. Sans surprise, on observe que les étudiantes et étudiants de la maîtrise sont moins nombreux à avoir participé à la production scientifique et à avoir été exposés à l'évaluation par les pairs.

\section{CONCLUSION}

La qualité de l'intégration académique est un déterminant important de la satisfaction des étudiantes et des étudiants de la maîtrise (profil recherche) et du doctorat à l'égard de leur expérience et, on peut le déduire, de la réussite des études. En particulier, c'est en étant exposés aux pratiques de recherche des autres et en débutant leurs propres projets de recherche, fussent-ils en partie définis par d'autres, que les étudiantes et étudiants développent les schèmes de perception et d'action propres au métier de chercheur dans leur domaine. Ils dépassent ainsi leur ancien rôle de consommateurs pour devenir des producteurs de nouvelles connaissances.

Ainsi, comme l'observe Lovitts (2001), la satisfaction des étudiantes et des étudiants aux cycles supérieurs est associée à leurs différents degrés d'accès aux ressources à la fois matérielles et intellectuelles requises pour l'apprentissage de la recherche, dont en particulier un financement approprié et une relation 
Tableau 4

Synthèse des variables explicatives du niveau de satisfaction globale des étudiantes et étudiants aux cycles supérieurs (écart-type entre parenthèses)

\begin{tabular}{|c|c|c|c|}
\hline \multicolumn{4}{|c|}{ Niveau de satisfaction (sur 4) } \\
\hline \multicolumn{2}{|c|}{ Hommes } & \multicolumn{2}{|c|}{ Femmes } \\
\hline \multicolumn{2}{|c|}{$3,15(0,75)$} & \multicolumn{2}{|c|}{$3,16(0,78)$} \\
\hline \multicolumn{4}{|c|}{ Nombre d'enfants } \\
\hline Aucun & \multicolumn{2}{|c|}{ Un } & Deux et plus \\
\hline $3,14(0,76)$ & \multicolumn{2}{|c|}{$3,39^{\mathrm{a}}(0,61)$} & $3,10(0,86)$ \\
\hline \multicolumn{4}{|c|}{ Domaine d'études } \\
\hline $\begin{array}{l}\text { Sciences naturelles et } \\
\text { génie }\end{array}$ & \multicolumn{2}{|c|}{ Sciences de la santé } & $\begin{array}{l}\text { Sciences humaines } \\
\text { et sociales }\end{array}$ \\
\hline $3,17(0,80)$ & \multicolumn{2}{|c|}{$3,09(0,71)$} & $3,18(0,75)$ \\
\hline \multicolumn{4}{|c|}{ Type de programme } \\
\hline \multicolumn{2}{|c|}{ Maîtrise } & \multicolumn{2}{|c|}{ Doctorat } \\
\hline \multicolumn{2}{|c|}{$3,09^{\mathrm{a}}(0,81)$} & \multicolumn{2}{|c|}{$3,23^{\mathrm{a}}(0,71)$} \\
\hline \multicolumn{4}{|c|}{ Source de financement (extrait) } \\
\hline $\begin{array}{l}\text { Bourse d'organisme } \\
\text { subventionnaire }\end{array}$ & $\begin{array}{c}\text { Aide financière } \\
\text { aux études (prêts et } \\
\text { bourses) }\end{array}$ & $\begin{array}{l}\text { Assistant de } \\
\text { recherche }\end{array}$ & $\begin{array}{c}\text { Auxiliaire } \\
\text { d'enseignement }\end{array}$ \\
\hline $3,29^{a}(0,76)$ & $3,00^{\mathrm{a}}(0,83)$ & $3,28^{a}(0,72)$ & $3,27^{\mathrm{a}}(0,68)$ \\
\hline
\end{tabular}

\begin{tabular}{ccc}
\hline \multicolumn{3}{c}{ Fréquence des rencontres avec le directeur } \\
\hline $\begin{array}{c}\text { Une fois par semaine } \\
\text { et plus }\end{array}$ & Deux-trois fois par mois & Une fois par mois \\
$3,25^{\mathrm{a}}(0,74)$ & $3,20^{\mathrm{a}}(0,76)$ & et moins \\
\hline
\end{tabular}

Perception de la progression dans le programme (étudiants)

\begin{tabular}{ccc}
\hline $\begin{array}{c}\text { À l'heure } \\
3,20(0,77)\end{array}$ & $\begin{array}{c}\text { En avance } \\
3,42^{\mathrm{a}}(0,71)\end{array}$ & $\begin{array}{c}\text { En retard } \\
2,90^{\mathrm{a}}(0,66)\end{array}$ \\
\hline \multicolumn{2}{c}{ Perception de la durée du programme (diplômés) } \\
\hline À l'heure & En avance & En retard \\
$3,42^{\mathrm{a}}(0,76)$ & $3,50(0,53)$ & $3,16(0,83)$ \\
\hline Au moins une & Aucune \\
\hline $3,19^{\mathrm{a}}(0,75)$ & $3,06^{\mathrm{a}}(0,80)$ \\
\hline Publications/communications évaluées par les pairs \\
\hline Au moins une & Aucune \\
$3,22^{\text {a }}(0,73)$ & $2,98^{\text {a }}(0,89)$ \\
\hline
\end{tabular}

a. Test t de Student significatif au risque de 5\%. 
enrichissante avec la directrice ou le directeur. Toutefois, la " ressource " qui apparaît la plus importante est un "bon " projet de recherche, c'est-à-dire un projet qui donne à l'étudiant l'impression d'avancer et de produire des résultats. Delamont, Parry et Atkinson (1997) soulignent d'ailleurs l'importance pour l'étudiant de travailler à un projet " faisable " (" do-able ") qui, malgré l'incertitude entourant toute entreprise scientifique, laisse envisager l'obtention de résultats significatifs. À plus forte raison, si ces résultats sont publiés, l'apprentiechercheuse ou l'apprenti-chercheur aura toutes les chances de se sentir reconnu par la communauté scientifique en tant que productrice ou producteur de nouvelles connaissances (Hagstrom, 1965). Au contraire, une étudiante ou un étudiant qui ne développerait pas de liens positifs avec, tout d'abord, sa directrice ou son directeur puis, plus largement, avec la communauté scientifique, a plus de chances d'être insatisfait de son expérience. Cette insatisfaction peut éventuellement mener à l'abandon des études, et ce d'autant plus lorsque la formation comporte des désavantages importants. Par exemple, on a vu que celles et ceux qui doivent recourir à l'aide financière aux études - et qui doivent ainsi s'endetter - sont nettement moins satisfaits que les autres étudiants. Au contraire, être encouragé par une bourse d'excellence ou un poste d'assistant de recherche ou d'enseignement peut accroître le sentiment d'être reconnu en tant qu'apprenti-chercheur et encourager la poursuite des études. À cet égard, la situation des étudiantes et étudiants de la maîtrise mériterait de faire l'objet d'une attention particulière, car leur socialisation à la recherche semble moins riche que celle caractérisant le doctorat. Tout en reconnaissant les contraintes spécifiques à la formation en maitrise, en commençant par l'inexpérience des étudiants euxmêmes, on peut se demander si certains ajustements pourraient être faits pour améliorer l'expérience de formation à la recherche au deuxième cycle.

Dans ce processus, la directrice ou le directeur joue un rôle essentiel et crucial, dont la marque peut se faire sentir sur toutes les autres dimensions de l'expérience étudiante. En effet, de par ses responsabilités d'encadrement, elle ou il doit s'assurer que le projet de recherche entrepris par l'apprenti est convenable et propre à mener à un produit - le mémoire ou la thèse - conforme aux normes en vigueur au sein du champ. Elle ou il joue également un rôle central dans la recherche de financement, que ce soit en offrant une bourse ou un salaire à même ses subventions de recherche, en écrivant des lettres de recommandation pour ses protégés ou simplement en enseignant l'importance des bourses d'excellence et la meilleure manière de monter un dossier convainquant pour en obtenir une.

L'accès à la publication est également contrôlé, consciemment ou non, par la directrice ou le directeur : les mécanismes par lesquels les articles sont préparés, soumis, évalués et publiés sont rarement connus des étudiants qui débutent en recherche. Ainsi, ce sera probablement à l'initiative de la personne qui les dirige que les étudiantes et étudiants feront leurs premières démarches de publication. Dans plusieurs disciplines, il s'agira d'ailleurs d'une publication conjointe professeur-étudiant, à laquelle pourront aussi collaborer d'autres 
membres de l'équipe de recherche.

Nous avons vu en introduction que certaines caractéristiques personnelles des étudiantes et des étudiants peuvent être associées à différents niveaux d'intégration, ce que nos données ne nous ont pas permis de vérifier. Au sujet du sexe des étudiants, en particulier, nous faisons l'hypothèse que cet effet, bien qu'invisible à l'échelle de l'ensemble de notre échantillon, varie dans les différentes disciplines en fonction de la proportion d'hommes et de femmes. Malheureusement, nous ne disposons pas pour le moment de catégories disciplinaires assez fines et d'un échantillon assez grand pour le vérifier. Une étude plus approfondie des rapports sociaux de sexe dans les universités francophones québécoises permettrait d'aller plus loin à ce sujet.

Dans un même ordre d'idées, nous avons été surpris de ne trouver aucune différence statistiquement significative dans le niveau de satisfaction globale des étudiantes et étudiants entre les groupes disciplinaires, alors que des taux de réussite très différents sont observés. Les modalités par lesquelles nous avons recruté les répondants de notre enquête, qui ont favorisé la participation d'un nombre élevé de personnes boursières des organismes subventionnaires, expliquent peut-être ce phénomène. En effet, une des causes habituellement avancées pour expliquer la plus faible réussite étudiante dans certains domaines est le défaut d'intégration académique : contrairement aux étudiants en sciences naturelles et en génie, ceux en sciences humaines et sociales sont moins présents à l'université, interagissent moins avec leurs pairs, ont moins accès à des postes d'assistant de recherche, etc. Or, nos méthodes de recrutement ont peut-être favorisé la formation d'un échantillon d'étudiantes et d'étudiants mieux intégrés. On y compte un nombre disproportionné de boursiers, notamment en sciences humaines et sociales. De plus, il est plausible que les étudiants qui ne sont pas très intégrés au monde académique - étant donc susceptibles d'être moins satisfaits - aient été moins portés à remplir un questionnaire portant sur une expérience dont ils se distancent de plus en plus. S'ils ne sont pas abonnés aux listes d'envoi de leur université, ne lisent pas les publications institutionnelles ou ne visitent pas souvent leur département, ils sont moins susceptibles d'avoir pris connaissance de l'existence de notre enquête.

Chose certaine, nos conclusions générales s'appliquent à toutes les disciplines : lorsqu'elles et ils disposent d'un financement adéquat, lorsque la relation avec leur directrice ou leur directeur est positive, et lorsqu'elles et ils ont l'impression de contribuer à la production de nouvelles connaissances, les étudiantes et étudiants ont davantage de chances d'être satisfaits de leur expérience de formation à la recherche.

Dans la mesure où la qualité de l'expérience de formation à la recherche et la réussite étudiante à la maîtrise et au doctorat sont des préoccupations pour les administrateurs universitaires et les membres du corps professoral, il convient de porter une attention accrue aux conditions de formation à la recherche, tant à l'échelle du département qu'à l'échelle individuelle. En plus de chercher à distribuer plus largement l'information sur les meilleures sources 
de financement et les manières d'y avoir accès, une attention accrue devrait être portée au processus de choix du sujet de recherche pour le mémoire ou la thèse, lequel est d'ailleurs inextricablement lié au choix de la directrice ou du directeur des travaux. Un encadrement accru du choix de sujet à la fois par la directrice ou le directeur et par les responsables du programme serait une manière de faire réaliser aux étudiantes et étudiants l'importance de cette étape et la pertinence de la réaliser tôt (Seagram, Gould \& Pyke, 1998). De telles mesures permettraient d'éviter un sentiment de piétinement dans l'avancement des études et amélioreraient les chances d'une progression rapide vers la production de nouvelles connaissances. Ceci n'exclut en rien la nécessité d'intégrer les savoirs antérieurs de la discipline. Au contraire, on peut même penser que, lorsque les étudiantes et étudiants ont identifié leur propre problème de recherche, l'acquisition des savoirs accumulés de leur champ prend davantage de sens parce que qu'ils sont désormais perçus à travers la perspective d'une problématique qu'ils se sont appropriée, et non d'un cadre simplement scolaire et strictement imposé de l'extérieur. On peut ainsi croire que, en encadrant un peu plus le processus de choix du sujet de recherche et en fournissant davantage de balises aux étudiantes et étudiants en la matière, on favorise leur cheminement vers l'acquisition d'un habitus de chercheur autonome.

\section{NOTES}

1. Le taux de sortants munis d'un diplôme ne doit pas être confondu avec le taux de diplomation au sein d'une cohorte donnée, considéré comme une mesure plus habituelle de la réussite dans les programmes. Cette dernière mesure n'est pas disponible pour le Québec à notre connaissance. Par ailleurs, les données disponibles ne permettent pas de distinguer les sortants d'une maîtrise dite " professionnelle " de ceux du profil " recherche ", le taux de réussite de ces derniers étant probablement moins élevé.

2. Comme l'indiquent Delamont, Parry et Atkinson (2001), même dans les cas où les étudiants sont inscrits à des programmes multi- ou interdisciplinaires, les chercheurs (aspirants ou accomplis) s'identifieraient surtout à une discipline unique, soit à leur discipline d'origine. Le cas des chercheurs ayant été initialement formés dans des programmes multidisciplinaires est plus complexe et poserait effectivement des problèmes de socialisation.

3. Nous avons en fait recueilli deux questionnaires de personnes ayant quitté leur programme avant d'en obtenir le diplôme, mais leur trop faible nombre nous empêche de les intégrer à l'analyse.

4. Le logiciel Plus ${ }^{2}$ de Le Sphinx Développement a été utilisé pour la conception et la mise en forme du questionnaire ainsi que pour la plupart des analyses présentées. Le questionnaire était mis en ligne de manière sécuritaire au moyen du service d'hébergement Sphinx0nline. Les questionnaires étaient protégés par un code d'accès individuel attribué à chaque répondant. Aucune information nominative au sujet des répondants n'a circulé par Internet en même temps que les réponses de ces derniers au questionnaire. 
5. Étant donné la très grande diversité de la structure des programmes de maîtrise et de doctorat d'une université à l'autre et d'une discipline à l'autre, nous avons préféré ne pas poser de questions détaillées sur les cours à suivre, les examens de synthèse ou les soutenances orales.

6. Trois phases de cueillette de données ont été effectuées. Une première version de l'enquête a été réalisée auprès des boursiers du programme de "Bourses de recherche en milieu de pratique " partagé par trois organismes subventionnaires québécois (le Fonds québécois de recherche sur la nature et la technologie, le Fonds québécois de recherche sur la société et la culture et le Fonds de recherche en santé du Québec) (Gemme, Gingras \& Milot, 2003). Ensuite, de légères corrections ont été apportées au questionnaire avant que ce dernier ne soit proposé aux inscrits du Régime de partenariat de l'Université de Sherbrooke (Gemme \& Gingras, 2003). Enfin, le questionnaire a été soumis à l'ensemble de la population étudiante aux cycles supérieurs des universités québécoises francophones (Gemme \& Gingras, 2005).

7. Nous avons utilisé le programme du congrès de l'Association francophone pour le savoir (Acfas) de mai 2002 pour identifier les chercheurs ayant réalisé une communication en collaboration avec une organisation nonuniversitaire. Nous avons aussi contacté les chercheurs universitaires ayant obtenu des contrats de recherche (identifiés grâce au Système d'information sur la recherche universitaire du Québec). Enfin, nous avons visité les sites Internet des Réseaux des centres d'excellence pour identifier les étudiants québécois qui y sont affiliés et qui sont ainsi susceptibles d'avoir des liens avec le milieu non-universitaire.

8. En 2003, presque autant d'hommes que de femmes étaient inscrits aux cycles supérieurs au Québec, soit $48 \%$ d'hommes et 52\% de femmes. Les femmes étaient légèrement plus nombreuses à la maitrise (53\%) et légèrement moins nombreuses au doctorat (46\%) (Ministère de l'Éducation du Québec, 2005).

9. En 2003, au Québec, les étudiants à temps partiel au doctorat comptaient un peu moins de $15 \%$. Cette proportion est nettement plus élevée à la maittrise (environ $55 \%$ ). Il faut garder à l'esprit que cette proportion comprend les étudiants inscrits aux programmes professionnels, et donc qui ne sont pas en formation à la recherche (Ministère de l'Éducation du Québec, 2005).

10. Seul un échantillon comportant un plus grand nombre de répondants avec deux enfants et plus, et éventuellement l'utilisation de méthodes qualitatives, permettrait de confirmer ce résultat et de l'interpréter de façon adéquate. 


\section{REMERCIEMENTS}

Nous remercions Olivier Bégin-Caouette, ayant effectué un stage au CIRST, et François Crevier-Lajeunesse, alors étudiant à la maitrise en sociologie à l'UQAM, pour leur participation à l'analyse des données présentées dans cet article. Olivier Craig-Dupont, alors étudiant au baccalauréat en Science, technologie et société de l'UQAM, et Véronique Lépine et Lionel Vécrin, alors qu'ils étaient respectivement étudiante à la maîtrise en histoire et étudiant à la maîtrise en sociologie à l'UQAM, ont également participé à plusieurs étapes de cette recherche. Merci aux évaluateurs anonymes qui ont fourni des recommandations détaillées et ont ainsi permis d'améliorer cet article. Enfin, Danielle Labbé a généreusement révisé notre article. Nous avons bénéficié du soutien financier du programme "Initiative de la nouvelle économie " du Conseil de recherche en sciences humaines du Canada et d'une subvention conjointe du Fonds québécois de recherche sur la nature et la technologie et du Fonds québécois de recherche sur la société et la culture. L'Université de Sherbrooke a également contribué financièrement à une partie de l'étude.

\section{RÉFÉRENCES}

Association canadienne pour les études supérieures (2003). L'Achèvement des études supérieures dans les universités canadiennes, rapport et recommandations. Ottawa : Association canadienne pour les études supérieures.

Becher, T., \& Trowler, P. R. (2001). Academic tribes and territories, second edition. Buckingham : Society for Research into Higher Education \& Open University Press.

Bowen, W. G., \& Rudenstine, N. L. (1992). In pursuit of the PhD. Princeton: Princeton University Press.

Conseil national des cycles supérieurs (2001). Les sources et modes de financement des étudiants aux cycles supérieurs. Montréal : Fédération étudiante universitaire du Québec.

Deem, R., \& Brehony, K. J. (2000). Doctoral students' access to research cultures - are some more unequal than others? Studies in Higher Education, 25(2), 149-165.

Delamont, S., Parry, O., \& Atkinson, P. (1997). Critical mass and pedagogic continuity: studies in academic habitus. British Journal of Sociology of Education, 18(4), 533-549.

Delamont, S., Parry, O., \& Atkinson, P. (2000). The doctoral experience. Success and failure in graduate school. London: Falmer Press.

Ehrenberg, R., \& Mavros, P. G. (1995). Do doctoral students' financial support patterns affect their times-to-degree and completion probabilities? The Journal of Human Resources, 30(2), 581-609. 
Gemme, B., Gingras, Y. \& Milot, P. (2003). Formation à la recherche et milieu de pratique: un portrait des boursiers du programme de Bourses de recherche en milieu de pratique. Note de recherche 2003-05. Montréal : Centre interuniversitaire de recherche sur la science et la technologie.

Gemme, B. \& Gingras, Y. (2003). La formation à la recherche en entreprise : Un portrait des étudiants et diplômés du "Régime de partenariat " de l'Université de Sherbrooke. Montréal : Centre interuniversitaire de recherche sur la science et la technologie.

Gemme, B. \& Gingras, Y. (2005). La formation à la recherche dans le cadre des collaborations université-milieu : analyse comparative des différents modes d'encadrement. Note de recherche 2005-04. Montréal : Centre interuniversitaire de recherche sur la science et la technologie.

Gingras, Y. (1991). L'institutionnalisation de la recherche universitaire et ses effets. Sociologie et sociétés, 23(1), 41-54.

Golde, C.M. (1998). Beginning graduate school: Explaining first-year doctoral attrition. New Directions for Higher Education, 101, 55-64.

Hagstrom, W.O. (1965). The scientific community. New York: Basic Books.

Kaufmann, J.-C. (2000). Ego : Pour une sociologie de l'individu. Paris : Nathan.

Legault, M. J.. (1997). L'effet de la division du travail sur la formation de nouveaux chercheurs. Cahiers de la recherche en éducation, 4(1), 27-58.

Lovitts, B. E. (2001). Leaving the ivory tower: The causes and consequences of departure from doctoral study. Lahnam : Rowman \& Littlefield.

Lovitts, B. E. (2005). Being a good course-taker is not enough: a theoretical perspective on the transition to independent research. Studies in Higher Education, 30(2), 137-154.

Ministère de l'Éducation du Québec. (2004). Indicateurs de l'éducation du Québec, édition 2004. Québec: Gouvernement du Québec.

Ministère de l'Éducation du Québec (2005). Statistiques de l'éducation. Enseignement primaire, secondaire, collégial et universitaire. Édition 2004. Québec : Gouvernement du Québec.

Pease, J. (1967). Faculty influence and professional participation of doctoral students. Sociological Inquiry, 37(Winter), 63-70.

Roth, W.-M. \& Bowen, G. M. (2001). 'Creative solutions' and 'fibbing results': Enculturation in field ecology. Social Studies of Science, 31(4), 533-556.

Seagram, B. C., Gould, J. \& Pyke, S. W. (1998). An investigation of gender and other variables on time to completion of doctoral degrees. Research in Higher Education, 39(3), 319-335. 
Seymour, E. \&t Hewitt, N. M. (1997). Talking about leaving: Why undergraduates leave the sciences. Boulder: Westview Press.

Tinto, V. (1993). Toward a theory of doctoral persistence. In Leaving college. Rethinking the causes and cures of student attrition. Second edition (pp. 230243). Chicago: University of Chicago Press.

\section{ADRESSE DE CORRESPONDANCE}

Centre interuniversitaire de recherche sur la science et la technologie

Université du Québec à Montréal

CP 8888 Succursale Centre-Ville

Montréal (Québec) H3C 3P8

gemme.brigitte@uqam.ca

Brigitte Gemme est étudiante au doctorat au département d'études sur l'éducation de l'Université de la Colombie-Britannique. Elle est également agente de recherche au Centre interuniversitaire de recherche sur la science et la technologie de l'Université du Québec à Montréal. Formée en Science, technologie et société ainsi qu'en sociologie, elle s'intéresse surtout aux politiques de la recherche, à la formation des scientifiques et des techniciens, et au rôle des universités dans la société. Sa thèse de doctorat porte sur la formation des chercheurs dans les champs scientifiques appliqués où les partenariats université-milieu sont courants.

Yves Gingras est professeur au département d'histoire de l'Université du Québec à Montréal (UQAM) et titulaire de la Chaire de recherche du Canada en histoire et sociologie des sciences. Ses travaux portent sur l'histoire et la sociologie des sciences et de l'enseignement supérieur. Il a récemment publié Éloge de l'homo techno-logicus, (Montréal, Fides, 2005), et, avec Brigitte Gemme, « L'emprise du champ scientifique sur le champ universitaire», Actes de la recherche en sciences sociales, no 164, septembre 2006, pp. 50-60; et « Formation professionnelle en milieu de pratique et insertion professionnelle des jeunes chercheurs ", dans L'insertion professionnelle des diplômés de cycles supérieurs, sous la direction de Wenceslas Mamboundou et Salim Laaroussi, Sainte-Foy, Presses de l'Université du Québec, 2005, pp. 59-72. 